\title{
Molecular diagnostics of hearing loss due to mutations in the SLC26A4 gene in indigenous peoples of Southern Siberia (Russia)
}

\author{
Valeriia Yuryevna Danilchenko \\ Institute of Cytology and Genetics \\ SB RAS, Novosibirsk, Russia \\ danilchenko_valeri@mail.ru \\ Ekaterina Alexandrovna Maslova \\ Institute of Cytology and Genetics \\ SB RAS, Novosibirsk, Russia \\ State University \\ Novosibirsk, Russia \\ maslova@bionet.nsc.ru
}

\author{
Marina Vyacheslavovna Zytsar \\ Institute of Cytology and Genetics \\ SB RAS, Novosibirsk, Russia \\ zytzar@bionet.nsc.ru \\ Olga Leonidovna Posukh \\ Institute of Cytology and Genetics \\ SB RAS, Novosibirsk, Russia \\ State University \\ Novosibirsk, Russia \\ posukh@bionet.nsc.ru
}

\author{
Marita Sergeevna Bady-Khoo \\ Perinatal Center of the Republic \\ of Tyva \\ Kyzyl, Russia \\ marita.badyhoo@mail.ru
}

\begin{abstract}
Establishing a genetic diagnosis of hearing loss is of great importance for clinical evaluation of deaf patients and for estimating recurrence risks for their families. Mutations in the SLC26A4 gene are a common cause of hearing loss in many regions of the world. This study presents the results of the first molecular genetic analysis of the $S L C 26 A 4$ gene sequence performed in patients with hearing loss of unknown etiology belonging to indigenous peoples of Southern Siberia - Tuvinians (the Tyva Republic) and Altaians (the Altai Republic). Contrast differences of the SLC26A4 pathogenic contribution to the etiology of hearing impairment were revealed: $28.2 \%$ for Tuvinians and $4.3 \%$ for Altaians. Both known and novel pathogenic variants as well as a wide range of polymorphic variants were found in the $S L C 26 \mathrm{~A} 4$ gene sequences of examined patients. High frequency of mutation c.919-2A $>G$ in Tuvinians is probably due to the founder effect.
\end{abstract}

Keywords - hereditary deafness, SLC26A4, molecular diagnostics, indigenous peoples of Southern Siberia

\section{Introduction}

Hereditary hearing loss is a rare monogenic disease with a unique high heterogeneity of genetic control. To date, about 160 genetic loci and 100 different genes associated with nonsyndromic hearing loss have been identified. Establishing a genetic diagnosis of hearing loss is of great importance for clinical evaluation of deaf patients and for estimating recurrence risks for their families. Mutations in the GJB2 gene have the most pathogenic contribution to development of isolated hearing loss in many populations, but there are significantly less data on the pathogenic contribution of other genes associated with hearing impairments. Mutations in the SLC26A4 gene (solute carrier family 26, member 4; 7q22.3, OMIM 605646) are the second most frequent cause of hereditary hearing loss, after mutations in the GJB2 gene, accounting for $\sim 10 \%$ of all hereditary hearing impairment cases at least in some Asian populations [1,2] making SLC26A4 gene testing essential for the establishment of genetic diagnosis of hearing loss.

The SLC26A4 gene consists of 21 exons and encodes transmembrane protein pendrin (780 amino acids) - a multifunctional anionic transporter that is mainly expressed in the inner ear, thyroid, and kidneys. Mutations in SLC26A4 lead to recessive nonsyndromic hearing loss (DFNB4, OMIM 600791) usually accompanied by the abnormalities in the inner ear structures such as the enlarged vestibular aqueduct (EVA) etc., which can be detected by computed tomography of the temporal bones, and some forms of Pendred syndrome (PDS, OMIM 274600) which combines hearing loss and goiter [3, 4].

At present, there is no sufficient experimental information on the pendrin structure and precise molecular mechanisms underlying function of this protein. Moreover, no systematic genotype-phenotype correlations have been made so far in deaf patients having hearing impairment presumably associated with $S L C 26 A 4$ mutations. Taking also into account a large physical size of the SLC26A4 gene, the development of routine SLC26A4 molecular diagnostics for hearing impairments is a nontrivial task.

About 260-500 pathogenic SLC26A4 variants associated with hearing impairments, according to different sources (ClinVar, https://www.ncbi.nlm.nih.gov/clinvar/; the Human Gene Mutation Database, http://www.hgmd.cf.ac.uk/ac/; the Deafness Variation

Database, http://deafnessvariationdatabase.org/), are currently revealed in patients in different populations worldwide but there are still many regions where the SLC26A4 contribution to deafness remains unknown.

Previously, we found that the proportion of deafness due to the mutations in the GJB2 gene in deaf patients belonging to indigenous peoples of Southern Siberia reaches to $22.3 \%$ among Tuvinian patients (the Tuva Republic) and 15.1\% among Altaian patients (the Altai Republic) [5]. However, the cause of hearing loss remains unclear for significant part of patients in these regions.

The aim of this study is to investigate the SLC26A4 pathogenic contribution in deafness in Tuvinian and Altaian patients with hearing loss of unknown etiology.

\section{MATERIALS AND METHODS}

Analysis of the SLC26A4 complete sequence including all 21 exons and flanking regions was performed by Sanger sequencing in the group of patients who were negative for the mutations in the GJB2 gene (171 out of 220 Tuvinian patients and 61 out of 93 Altaian patients). To investigate the inner ear abnormalities, computed tomography of the temporal bones (CT) was conducted in patients with detected SLC26A4 mutations. Analysis of the carrier frequency of SLC26A4 
mutations was performed in the ethnically matched samples of unrelated healthy individuals (157 Tuvinians, 218 Altaians). Several bioinformatics prediction tools (PolyPhen2; PROVEAN; FATHM; MutationAssessor; etc.) were applied for evaluation of pathogenicity of novel SLC26A4 variants. Two-tailed Fisher's exact test with significance level of $p<0.05$ was applied to compare allele frequencies between patients and controls.

\section{RESULTS}

A molecular genetic analysis of the SLC26A4 gene was performed in patients with hearing loss of unknown etiology belonging to indigenous peoples of Southern Siberia: Tuvinians (the Tyva Republic) and Altaians (the Altai Republic). Both known: c.170C $>$ A (p.Ser57Ter), c.919$2 \mathrm{~A}>\mathrm{G}, \quad$ c.2027T $>\mathrm{A}$ (p.Leu676Gln), c.2034+1G $>\mathrm{A}$, c.2168A $>\mathrm{G}$ (p.His723Arg) and novel (c.1545T $>\mathrm{G}$ (p.Phe515Leu), c.1717G $>\mathrm{T}$ (p.Asp573Tyr) pathogenic recessive variants as well as a wide range of benign variants were found in the SLC26A4 sequences of patients. Probable pathogenicity of novel SLC26A4 variants was established by bioinformatics prediction tools, and for variant c.1545T $>\mathrm{G}$ was confirmed by its segregation with hearing loss revealed by the analysis of patient's pedigrees. Significantly higher pathogenic contribution of the SLC26A4 gene in deafness (estimated by the presence of biallelic recessive SLC26A4 mutations) was found in Tuvinian patients $(28.2 \%)$ in contrast to Altaian patients $(4.3 \%)(\mathrm{p}<0.05)$ (Table 1$)$.

TABLE I. SLC26A4 GENOTYPES FOUND IN EXAMINED PATIENTS

\begin{tabular}{|c|c|c|}
\hline$S L C 26 A 4$ genotypes & $\begin{array}{l}\text { Tuvinian } \\
\text { patients }(n=220)\end{array}$ & $\begin{array}{l}\text { Altaian patients } \\
(\mathrm{n}=93)\end{array}$ \\
\hline \multicolumn{3}{|c|}{ Homozygous and compound heterozygous SLC26A4 genotypes } \\
\hline c. $[919-2 \mathrm{~A}>\mathrm{G}] ;[919-2 \mathrm{~A}>\mathrm{G}]$ & 30 & - \\
\hline c. $[2027 \mathrm{~T}>\mathrm{A}] ;[2027 \mathrm{~T}>\mathrm{A}]$ & 4 & - \\
\hline c. $[2168 \mathrm{~A}>\mathrm{G}] ;[\mathrm{c} .2168 \mathrm{~A}>\mathrm{G}]$ & - & 2 \\
\hline c. $[170 \mathrm{C}>\mathrm{A}] ;[170 \mathrm{C}>\mathrm{A}]$ & 1 & - \\
\hline c. $[919-2 \mathrm{~A}>\mathrm{G}] ;[2027 \mathrm{~T}>\mathrm{A}]$ & 14 & 2 \\
\hline c. $[919-2 \mathrm{~A}>\mathrm{G}] ;\left[1545 \mathrm{~T}>\mathrm{G}^{*}\right]$ & 8 & - \\
\hline c. $[170 \mathrm{C}>\mathrm{A}] ;[919-2 \mathrm{~A}>\mathrm{G}]$ & 3 & - \\
\hline c. $[919-2 \mathrm{~A}>\mathrm{G}] ;[2034+1 \mathrm{G}>\mathrm{A}]$ & 1 & - \\
\hline c. $\left[1545 \mathrm{~T}>\mathrm{G}^{*}\right] ;[2027 \mathrm{~T}>\mathrm{A}]$ & 1 & - \\
\hline Total & $62(28.2 \%)$ & $4(4.3 \%)$ \\
\hline \multicolumn{3}{|l|}{ Heterozygous $S L C 26 A 4$ genotypes } \\
\hline c. $[919-2 \mathrm{~A}>\mathrm{G}] ;[?]$ & 9 & - \\
\hline c. $[170 \mathrm{C}>\mathrm{A}] ;[?]$ & 1 & - \\
\hline c. $\left[1545 \mathrm{~T}>\mathrm{G}^{*}\right] ;[?]$ & 1 & - \\
\hline c. $[2027 \mathrm{~T}>\mathrm{A}] ;[?]$ & 1 & 1 \\
\hline c. $\left[1717 \mathrm{G}>\mathrm{T}^{*}\right] ;[?]$ & - & 1 \\
\hline Total & 12 & 2 \\
\hline
\end{tabular}

* - novel variant
Additional investigation of the SLC26A4 gene sequence is necessary to perform in group of deaf patients with monoallelic SLC26A4 mutations (Table 1). The enlargement of vestibular aqueduct (EVA) (varying from $1.5 \mathrm{~mm}$ to 5.1 $\mathrm{mm}$ ) was revealed in most patients homozygous or compound heterozygous for SLC26A4 mutations who passed CT $(\mathrm{n}=27)$. To note, the degree of EVA was characterized by sufficient intra and interfamily variability.High frequency of mutation c. $919-2 \mathrm{~A}>\mathrm{G}$ in Tuvinians $(69.9 \%$ of all mutant alleles detected in patients and carrier frequency reaching to $5.1 \%$ in Tuvinian control sample) is probably due to the founder effect.

\section{CONCLUSION}

This is the first study to address the SLC26A4 mutations contribution in deafness in indigenous populations of Southern Siberia. Contrast differences in the proportion of deafness caused by the SLC26A4 mutations were revealed in Tuvinians and Altaians despite of related ethnicity and geographically close territories of residence of these indigenous peoples of Southern Siberia. Novel data on spectrum and prevalence of pathogenic and benign variants of gene SLC26A4 in Siberian populations significantly contribute to the SLC26A4 allelic diversity worldwide.

\section{ACKNOWLEDGMENTS}

Study was supported by the Projects \#0259-2019-0010-C01, \#0324-2019-0041-C-01, and the RFBR grant \#17-2906016_ofi-m.

\section{REFERENCES}

[1] K. Tsukamoto, H. Suzuki, D. Harada, A. Namba, S. Abe, S. Usami. Distribution and frequencies of PDS (SLC26A4) mutations in Pendred syndrome and nonsyndromic hearing loss associated with enlarged vestibular aqueduct: a unique spectrum of mutations in Japanese. Eur J Hum Genet. 2003, vol. 11(12), pp. 916-922.

[2] Q. J. Wang, Y. L. Zhao, S. Q. Rao, Y. F. Guo, H. Yuan, L. Zong, et al. A distinct spectrum of SLC26A4 mutations in patients with enlarged vestibular aqueduct in China. Clin Genet. 2007, vol. 72(3), pp. 245254.

[3] N. Chen, L. Tranebjærg, N. D. Rendtorff, I. Schrijver. Mutation analysis of SLC26A4 for Pendred syndrome and nonsyndromic hearing loss by high-resolution melting. J Mol Diagn. 2011, vol. 13(4), pp. 416-426.

[4] L. A. Everett, B. Glaser, J. C. Beck, J. R. Idol, A. Buchs, M. Heyman, et al. Pendred syndrome is caused by mutations in a putative sulphate transporter gene (PDS). Nat Genet. 1997, vol. 17(4) pp. 411-422.

[5] O. L. Posukh, M. V. Zytsar, M. S. Bady-Khoo, V. Y. Danilchenko, E. A. Maslova, N. A. Barashkov, et al. Unique mutational spectrum of the GJB2 gene and its pathogenic contribution to deafness in Tuvinians (Southern Siberia, Russia): a high prevalence of rare variant c.516G $>$ C (p.Trp172Cys). Genes (Basel). 2019, vol. 10(6) p. 429. 